\title{
College-aged Men's (18-24) Knowledge and Perceptions of Human Papillomavirus and Cervical Cancer
}

\author{
Ricardo V. López, Sora Park Tanjasiri, Shari McMahan \\ California State University, Fullerton
}

\begin{abstract}
College-aged men (18-24) can easily transmit HPV, the most common type of sexually transmitted infection in the United States, but have the lowest knowledge of HPV and cervical cancer. This study utilized the Health Belief Model to assess the knowledge and perceptions of HPV and cervical cancer among college-aged men, and their intent to reduce their numbers of future sex partners and wear condoms during their next sexual encounter. Of 100 participants, one third $(33.7 \%)$ had never heard of HPV before this study, and $87.9 \%$ rated their subjective knowledge of HPV knowledge as "poor". Participants reported high perceived severity, and susceptibility to, HPV infection. Having heard of HPV, being in a relationship with a female partner and self-efficacy to wearing condoms were associated with participants' intent to wear condoms during their next sexual encounter. Perceived benefits were associated with participants' intent to reduce the number of future sex partners. College-aged men should be targeted for HPV risk reduction with HPV health interventions tailored to augment male involvement in HPV and cervical cancer prevention efforts.
\end{abstract}

(C) 2008 Californian Journal of Health Promotion. All rights reserved.

Keywords: Human Papillomavirus, S T I, Cervical Cancer, College-aged Men, Self-Efficacy

\section{Introduction}

Human papillomavirus (HPV), a sexually transmitted infection, accounts for over $90 \%$ of all cervical cancer cases (Koutsky, 1997; Schiffman \& Castle, 2003). The highest rates of HPV infection are found among adults 18 to 28 years old (Koutsky, 1997). Within that group, both men and women in the 20-to 24-year age group have been found to be at higher risk for HPV infection (Burk, Ho, Beardsley, Lempa, Peters, \& Bierman, 1996). Cervical infections, with high-risk HPVs (HPV 16 and 17), begin with genital to genital, hand to genital, and oral to genital contact and can lead carcinogenesis (Schiffman \& Castle, 2003). At first HPV causes low grade squamous intraepithelial lesions (LSILs) or high grade squamous intraepithelial lesions (HSIL) (Franco, Schlecht, \& Saslow, 2003). Both types of lesions can occur independently, but LSILs can also progress to HSIL. Women with invasive cervical cancer average 50 years of age and women with HSIL average 28 of age; thus suggesting a long time period before progression to carcinogenesis. In advanced stages treatment is very expensive and almost ineffective despite of it being one of the most preventable cancers in the United States. In 2003, there were approximately 11,892 new cases of cervical cancer and 3,850 deaths due to the disease (Baseman \& Koutsky, 2005).

\section{HPV among the college-aged population}

Studies of college-aged students and HPV are relatively few, and are mainly focused on women. They show that women lack sufficient knowledge, have misperceptions about their susceptibility to HPV, and are unaware of the risk factors that increase the likelihood of infection (Lambert, 2001; Yacobi, Tennant, Ferrante, Pal, \& Roetzhiem, 1999). Research demonstrates that $10 \%$ to $39 \%$ of sexually active young women, especially those of college age (18-24), are infected with high risk HPV at any point in time (Auvinen, Niemi, Malm, Zilliacus, Trontii, Fingerrroos et al., 2005; Shin, Franceschi, Vaccarella, Roh, Ju, Oh et al., 2004; Winer, Lee, Hughes, Adam, Kiviat, \& Koutsky, 
2003). Potential interventions to decrease the likelihood of HPV infections in this population of women should consider the role men play in HPV infection; men are proven to be the primary carriers and vectors in the epidemiological triangle of HPV infection (Castellsague, Bosch, \& Munos, 2001; Franceschi, Castellsague, Del Maso, Smith, Plummer et al., 2001). In addition, most HPV infections in men are asymptomatic; thus men generally have sub-clinical HPV infection (Castellsague et al., 2001; Hippelainen, Syrjanen, \& Hippelainen, 1993). Studies show that sexually active college-aged men possess substantial rates of HPV infection ( $10 \%$ to $45 \%)$ at any point in time (Franceschi et al., 2002; Lazcano-Ponce, Herrero, Munoz, HernandezAvila, Salmeron, Leyva et al., 2000; Shin et al., 2004; Svare, Kjaer, Worm, Osterling, Meijer \& Van den Brule, 2006; Wikstrom, Popescu \& Forsland, 2000). Elucidating cognitive factors in college-aged men may help reduce the incidence of HPV infection among college-aged women. Curtailing the incidence of HPV in women at younger ages may help decrease the percentage of women that develop cervical cancer later in life (Baseman \& Koutsky, 2005).

\section{The Health Belief Model approach towards HPV prevention}

The Health Belief Model (HBM), a value expectancy model, was used to guide the development of this study's instrument. The HBM contends that preventative action is influenced by an individual's perceived susceptibility to the disease, perceived severity to the disease, perceived benefits of reducing risk to the disease, and perceived barriers to taking preventative action (Glanz, Lewis, \& Rimer, 2002). Self-efficacy, defined as one's self-judgments of personal capabilities to initiate and successfully perform specified tasks at designated levels, expend greater effort, and persevere in the face of adversity, was also included in the HBM (Bandura, 1986). Evidence shows that self-efficacy plays a central role in the prediction of initiationand maintenance of healthy behavior changes (Glanz et al., 2002).

Several studies on other STIs (primarily HIV) have operationalized one to multiple constructs, and in some cases all of the HBM. Zak-Place and Stern (2004) examined the full application of the HBM and self-efficacy, with regard to STD and HIV preventive behavioral intentions in female and male college-aged students. Selfefficacy was identified as the primary predictor of behavioral intentions in regards to condom use, STD testing and HIV testing. They concluded, however, that the use of the full HBM in predicting college students' sexual preventative behavior is unsupported. In another study, Ingledue and colleagues (2004) were able to find in a population of college-aged women that: (1) as HPV knowledge increased, perceived seriousness decreased; (2) as number of sexual partners increased, perceived susceptibility to $\mathrm{HPV} /$ cervical cancer increased; and (3) those that perceived and felt susceptible to HPV/cervical cancer were more like to receive a Pap test in the past year (Ingledue et al., 2004). Lopez and McMahan (2007) also applied the full HBM to examine college women's perceptions of HPV and cervical cancer, and to predict their intent to practice preventative sexual behaviors. They found that only selfefficacy is useful in predicting intentions of carrying out preventative sexual behavior.

\section{The present study}

Fewer studies have included only young adult males in their exploration of the knowledge and perceptions of HPV and cervical cancer in college-aged students (Lambert, 2001; Yacobi et al., 1999). In the only known study of collegeaged males' perceptions and knowledge of HPV and cervical cancer, McPartland and colleagues (2005) found that men who had knowledge of HPV and its links to cancer had higher intentions of reducingtheir number of sex partners, and use a condom with a new partner. This suggests that illuminating a clearer picture of college-aged men's knowledge and perceptions of HPV and cervical cancer may help in the formulation of prevention interventions that may reduce HPV infection rates in women (Castellsague et al., 2001; Munoz \& Bosch, 1997). Thus, the purpose of this study was to explore the knowledge and perceptions of HPV infection and cervical cancer among college-aged men, and to examine their intent to reduce their numbers of sex partners and wear condoms during their next 
sexual encounter. Participants' intent to wear condoms the next time they have sex and to reduce their number of future sexual partners to prevent HPV infection were assessed through the HBM framework. The HBM was expected to provide a more holistic look at cognitive measures that affect college-aged men's behavior with regards to HPV and cervical cancer.

It was expected that participants with lower perceived severity to HPV infection would not intend to reduce their number of sex partners in the future and would not intend to wear a condom the next time they have sex. Furthermore, ir was predicted that men with lower perceived susceptibility to HPV infection would not intend to reduce their number of sex partners in the future and would not intend to wear a condom the next time they have sex. Conversely, it was predicted that men with high perceived barriers to preventing HPV infection would not reduce their number of sex partner(s). It was also anticipated that men with low perceived benefits to preventing HPV infection would not intend reduce their number of future sex partner(s) and wear a condom the next time they have sex. It was expected that men will have low self-efficacy to wear condoms and would not intend to wear condoms the next time they have sex with their female partner(s). Lastly, it was expected that men will have low knowledge of HPV and cervical cancer.

\section{Methods}

\section{Participants}

A cross-sectional questionnaire of knowledge, perceptions and behavioral intentions was distributed to college-aged men attending elective personal health classes (in 2006 through the department of Health Science), ata public university in Southern California. . The student population of 37,000 at this university was highly diverse (32\% White, 28\% Hispanic, 22\% Asian Pacific Islander, 4\% Black, 16\% other/unknown) and slightly exceeded the ethnic diversity found in the state of California (47\% White, 32\% Hispanic, 11\% Asian Pacific Islander, 7\% Black, 1\% American-Indian). The classes involved in this study ranged in size from 25 to 125 students with mixed representation of ethnicities, majors, and class levels; about $30 \%$ of the students were men. All men aged 18 who were heterosexual, or men who have sex with men (MSM) and all women were eligible to participate in this study. Questionnaires from MSM and women were omitted during data analysis. Men over the age of 24 do not comprise a significant portion of the college-aged population in most California State Universities; thus their questionnaires were also omitted during data analysis. All protocols for participant selection and participation were approved by the Institutional Review Board (IRB) on campus prior to implementation.

\section{Measures}

Participants took a modified version of the selfadministered questionnaire called the Knowledge and Perceptions Survey (KAPS). The KAPS instrument was created by McPartland and colleagues (2005) to assess perceived severity, susceptibility, knowledge of HPV, and intent to practice HPV preventative health behavior. Questions to examine knowledge of the existence of the HPV vaccine, perceived barriers, perceived benefits and selfefficacy to wear condoms were incorporated in KAPS for the purpose of this study. In addition, participants' number of sexual partners in the last 12 months and whether they were in a heterosexual relationship during that time period were also assessed. We also asked whether the reported relationship was monogamous. Participants were asked questions about their intent to wear condoms (the next time they have sex) and intent to reduce their number of future sexual partners in order prevent HPV infection. Demographics examined included ethnic background, age, class standing (freshman, sophomore, junior or senior), and whether the student lived at home or independently. Cronbach's Alpha was calculated for the 13item knowledge scale. Face validity of the questionnaire was assessed by four health science faculty and two health professionals from two public universities in the Southern California area. The instrument was pilot tested with five males from the priority population to establish questionnaire logistics, time frame, skip patterns and replace vague or confusing 
questions. Revisions were made based on feedback from the pilot study.

There were two outcome variables in this study: participants' intent to wear a condom during their next sexual encounter, and participants' intent to reduce the number of future sexual partners to prevent HPV infection. There were six main predictor variables; each of the five HBM constructs, and the participant's score on the cervical cancer/HPV knowledge scale. Participants' score on the HPV/cervical knowledge scale was based on 13 true or false questions. The predictor and outcome variables contained4-point Likert scale.

Others variables that were assessed and treated as predictor variables, were: participant's ethnic background, number of sexual partners in the last 12 months, whether they were in a heterosexual relationship and if that relationship was monogamous, whether they were sexually active in the last 12 months, if they lived independently or were dependent upon a parent/ guardian, if they used a condom during their last sexual encounter and if they use condoms with their current sexual partner(s).

\section{Participant Recruitment}

A letter and e-mail were sent to instructors from all personal health general education courses during the fall 2006 semester requesting class time to distribute the questionnaires during the last ten minutes of a pre-determined class lecture. After obtaining instructor consent, the first author presented the study to students at the end of each class time. Students were verbally notified of their rights to refuse, and provided instructions to fill out the anonymous questionnaires. Next, all students (male and female) 18 years and older in attendance were given the self-administered questionnaire. Special protocols were followed to increase the anonymity and comfort level of potential participants. Participants were asked to place all anonymous questionnaires in a box located at the front of the classroom. The exact number of male and female students in attendance was obtained to calculate participation rates.

\section{Data Management and Analyses}

Cronbach's $\alpha$ was calculated for the 13-item knowledge scale. Participants' mean age was calculated, as well as frequencies for class standing and ethnicity.Ethnic background was recoded into a categorical variable: MiddleEastern (1), White-non-Hispanic (2), Asian/Pacific Islander (3), Black/AfricanAmerican (4), American Indian (5), Latino/Hispanic being (6) and "other" was recoded as 7. The ethnic category of "other" comprised those who were of mixed/multiple ethnicities or reported an ethnic background not reflected in the available options.

Other information analyzed for possible confounding effects included the following (and were given 1 for "yes" and 0 for "no" unless otherwise stated): if the participant reported being in a relationship and if that relationship was monogamous, if the participant lived with parents/guardians, if the participant lived independently without the presence of parents/guardians, the number of sexual partners in the last 12 months ( 1 for those that reported 1 or more, and zero for those with no partners), if the participant had ever heard of HPV, subjective knowledge of HPV ( 1 for good and 0 for poor), if they used a condom during their last sexual encounter, and if they use a condom with their current sexual partner(s).

Each participant received one point for each question answered correctly and zero for incorrect answers in the knowledge scale. The knowledge score was then converted into a dichotomous low (i.e., below the mean) vs. high (i.e., above the mean) knowledge variable as in McPartland and colleagues (2005) and Yacobi and colleagues (1999). All constructs of the HBM were turned into dichotomous variables for the purpose of statistical analysis as done in McPartland and colleagues (2005). Outcome variables were also dichotomized for the purpose of data analysis. For intent to reduce sex partners the answers "not at all likely" and "fairly likely" were recoded as 0 (no intention to reduce sex partners) and answers "very likely" and "definitely likely" were recoded as 1 for each variable (intent to reduce sex partners). For 
intent to wear condoms the next time participants have sex the answers "not at all likely" and "fairly likely" were recoded as 0 (no intention to wear condoms) and answers "very likely" and "definitely likely" were recoded as 1 for each variable (intent to wear condoms).

Unadjusted logistic regression analysis was performed to assess the relationship between the individual predictor variables with each outcome variable. Predictor variables with a $p$-value less than 0.20 were then included in an adjusted multivariate logistic regression model. All appropriate statistical analyses were conducted with the Statistical Package for the Social Science (SPSS) software, version 14.0 (SPSS Inc., Chicago, IL)

\section{Results}

Of the 128 male students who were invited to participate in the study, 113 completed a survey for a participation rate of $88.3 \%$. Of the 113 male surveys, 13 were excluded for the following reasons: Five participants were over the age of 24 , five did not report a date of birth, two were self-identified MSM and one answered only the demographic questions of the questionnaire. The final sample included 100 college-aged men. As shown in Table 1, the ethnic distribution was diverse, and closely mirrored the ethnic distribution of the university in question in 2006; nearly half were freshmen; and nearly all were full-time students who lived at home. Over half were in a relationship with a significant other, with one third reporting that this relationship was monogamous. Thirty-two percent $(32 \%)$ stated that they did not have any sexual partners in the last 12 months. Forty-two percent (42\%) were sexually active; $32 \%$ stated that they had at least one partner in the last 12 months, and $36 \%$ had more than one partner during that same time period. The majority $(56.6 \%)$ reported using condoms with nearly two-thirds $(61.9 \%)$ reporting that they used condoms every time they had sex.

\section{College Age Men's Knowledge of HPV and Cervical Cancer}

As shown in Table 2, about one third (33.7\%) of men had never heard of HPV before this study.
Those that heard of HPV heard it primarily from media outlets (television/radio, internet, newspaper/magazines), but also from family/friends, health care providers, classes, and from their sexual partner(s). Almost 88\% $(87.9 \%)$ rated their subjective knowledge of HPV as "poor" and only $12.1 \%$ thought their knowledge was "good". Nearly all men knew that having one type of HPV did not mean that they could not acquire a new type (81\%), that they can transmit HPV even without symptoms (79\%), and that most people with HPV have no signs or symptoms $(75 \%)$. Fewer men, however, knew that HPV does not cause herpes (25\%), that there is an HPV vaccine for women $(36.0 \%)$, and that HPV can cause genital warts (44\%). Overall, $23 \%$ of the participants obtained six questions correct or less and $77 \%$ obtained seven or more questions correct on the 13 item scale $($ Mean $=7.93$, Median $=8.00, \mathrm{SD}=2.00)$. Participants' scores on individual questions are noted on Table 2. The internal consistency of the knowledge scale was $\alpha=0.38$.

\section{College Age Men's Perceptions of HPV Infection}

Participants reported high perceived severity of (74\%), and susceptibility to $(88.9 \%)$ HPV infection. They also reported perceived benefits $(75.3 \%)$ to preventative sexual behavior and perceived obstacles $(81.4 \%)$ to initiating those behaviors. Of those who were sexually active, $74.3 \%$ were self-efficacious to wear condoms during their next sexual encounter, 26\% intended to wear condoms the next time they have sex, and $53.4 \%$ intended to reduce their future number of sex partners in order to decrease the likelihood of contracting HPV.

\section{Unadjusted Odd Ratios}

As shown in Table 3, perceived severity, perceived obstacles, perceived susceptibility, knowledge of HPV, subjective knowledge of HPV, living independently, and living at home were not statistically significant at the $p=0.05$ level. Ethnicity, status of sexual activity and number of sexual partners in the last 12 months were also not statistically significant. Black/African-American and Middle-Eastern participants were not included in logistic regression analyses due to their low frequencies. 
Men with perceived self-efficacy to wear condoms were 24.44 times as likely to have intentions of wearing a condom the next time they had sex to prevent HPV infection (95\% CI = 6.13-97.48); men who had heard of HPV infection were 13.80 times as likely to have intentions of wearing condoms the next time they had sex (95\% CI $=1.71-111.09)$. In addition, men who reported being in a relationship with a female partner where 3.25 as likely to have intentions to wear a condom during their next sexual encounter $(95 \% \mathrm{CI}=$ 1.03-10.28). With regards to number of sex partners, men with perceived benefits to reducing sex partners were 3.77 times as likely to have intentions of reducing future sex partners to prevent $\mathrm{HPV}$ infection $(95 \% \mathrm{CI}=$ $1.16-12.25)$

\section{Adjusted Odd Ratios}

The following variables were included in an adjusted model to predict intent to wear condoms, because they were statistically significant in the univariate analyses: being in a relationship, having heard of HPV and selfefficacy to wear a condom to prevent HVP infection. Men who reported high self-efficacy were 40.45 times as likely to have intentions of wearing a condom the next time they had sex $(95 \% \mathrm{CI}=6.49-252.32)$. Men who had heard of HPV were 26.21 as likely of intending to wear a condom the next time they had sex $(95 \% \mathrm{CI}=$ 1.84-373.82). In addition, men who were in a relationship were 3.18 as likely of intending to wear a condom the next time they have sex $(95 \%$ CI $=0.51-19.74)$. No adjusted odds ratios were calculated for the outcome variable intent to reduce number of future sex partners since only the perceived benefits variable was significantly associated with predictor variables at the univariate level of analyses.

\section{Discussion}

In our study, college-aged men had a higher awareness of HPV compared to past studies (McPartland et al., 2005; Yacobi et al., 1999). Men reported hearing of HPV through a myriad of sources, but overwhelmingly through media outlets such as TV, radio, newspapers, magazines and the internet. This is likely due to media campaigns advocating for the widespread availability of a vaccine that prevents most virulent types of HPV infection in women. None had heard of HPV through college mediated health promotion campaigns. Those who had heard of HPV $(66 \%)$ were more likely to intend to reduce the number of future sex partners. However, as Yacobi and colleagues (1999) suggest, we suspect that this intent may be due to students' familiarity with HIV. HIV education tends to overshadow HPV education on college campuses (Yacobi et al., 1999). College based health centers should do more to publicly expose men to the existence of HPV and cervical cancer separately from HIV/AIDS. Given the high influence of the internet, they could advertise about HPV/cervical cancer on their university/college websites. Subsequent interventions could focus on increasing men's perceived knowledge of HPV, by attaching short quizzes within website advertisements that highlight key factors of an HPV infection.

In this study, as in the study by McPartland and colleagues (2005), knowledge was not significantly associated with intent to carry out preventative sexual behaviors. Yacobi and colleagues (1999) had found an association between knowledge and participants' ability to take additional precautions to prevent HPV infection. They suggested that knowledge is an important tool in altering health behaviors. Participants of Yacobiet al., , however, were both female and male. Participants in this study, as well as in McPartland et al.'s study, were all male. Men may perceive HPV infection and cervical cancer as women-only oriented issues, and therefore may not be motivated to initiate preventative health behaviors.. This attitude may be supported by the fact that public and private health promotion HPV campaigns are overwhelmingly targeted towards women. This gender apathy is evidenced by participants' low score on knowledge questions that were specific to HPV infection in women. Future campusbased health promotion campaigns should increase efforts to deliver HPV health education interventions tailored to college-aged men, given their pivotal role in women's HPV infection. Such programs may reduce male promiscuity 
and incite other STI interventions to support male-based preventative health education.

McPartland and colleagues (2005) indicated that men's intent to reduce their number of sex partners was significantly associated with their perception of HPV severity to their female partner(s). We found that men may be more likely to carry out intended health behavior modifications if benefits relate directly to them, . Research that supports the relationship between perceived benefits and intended behavior modification in college-aged men is not extensive. Zak-Place and Stern (2004) suggested that examining perceived benefits is not conducive to predicting risk reduction, but their study was done on a population of female students. Another study suggested that the perceived benefits construct is a strong predictor of preventive sexual behavior, but also with a population of women (Cecil, Pinkerton \& Bogart, 1999). We suggest that college health promotion campaigns should also emphasize health preventative factors, such as condom use, that are mutually beneficial for men and their partners.

With risk reduction, differences were found by preventative health behavior. Condoms are a primary method of preventing STIs in sexually active individuals, yet less than half of all college students report using condoms consistently (Zak-Place \& Stern, 2004). In our study, only $43 \%$ of sexually active men wore a condom during their last sexual encounter, and only $26 \%$ intended to wear condoms the next time they have sex. More efforts to promote condom wearing behavior are important. Although most men in this study felt it would be difficult to reduce their number of sexual partners for the sake of preventing HPV infection, over half felt confident in their ability to wear condoms. Wearing condoms may not prevent all types of anogenital HPV infections, but will reduce the likelihood of transmission (Schiffman \& Castle, 2003). Also, men who were in a relationship were more likely to intend to wear a condom during their next sexual encounter. As McPartland and colleagues (2005) alluded, men's relationship status with their female partners may be more influential than any other variable in increasing their intent to practice preventative sexual behavior. Collegebased health promotion campaigns should further examine the role of feelings and affection between college-aged men and their female partner(s). They should also examine if there is a relationship between monogamy and college-men's intentions to practice preventative sexual behavior.

Skills building should also be considered in future prevention programs. Similar to ZakPlace and Stern (2004), we found self-efficacy to be a significant predictor of intent to use condoms as an HPV preventative health behavior. Intent to wear condoms is a long-term behavior change, and is something that sexually active college-aged men must re-evaluate before every sexual encounter. Evidence shows that self-efficacy goes beyond predicting simple behavior and plays a central role in the prediction of initiation and maintenance of longterm healthy behavioral changes (Glanz, et al., 2002). College health promotion campaigns designing interventions on HPV preventative health behavior should create/enhance health educational experiences that augment males' condom wearing skills. Self-efficacy to wear condoms may be increased by creating innovative ways of teaching college-aged men how to properly store and put on a condom. This could boost the initiation and maintenance of proper condom wearing behavior to reduce HPV infection. More male HPV prevention may lower rates of female HPV infections on college campuses, and subsequently lead to reduced rates of cervical cancer later in time.

\section{Limitations}

Given the exploratory nature of this study, findings may be limited by many factors. First, the cross-sectional design cannot establish cause and effect between independent and dependent variables. In addition, only intent was measured and there was no verification of actual behavior modifications. Furthermore, the use of a selfadministered questionnaire may have introduced social desirability. Since the questionnaire was handed out in a classroom setting, participants may have had trouble dealing with issues of confidentiality, thus limiting the truthfulness of their responses. Additionally, a larger sample 
may have allowed for a more robust test of the hypotheses.

In addition, the modified KAPS questionnaire was not created to be a full comprehensive assessment tool, since only one question was asked to measure each construct of the HBM. McPartland and colleagues (2005) also did not report any original validity and reliability estimates for the KAPS or the 13 item knowledge scale. Despite its low internal consistency in this study, the knowledge scale and the modified KAPS were still used for the purpose of hypothesis testing due to the novelty of this realm of research. Future studies should undertake a more comprehensive assessment of theoretically-derived constructs. For example, more focus should be placed on developing a detailed HPV knowledge scale that may be more indicative of preventative sexual behavioral intent in men. Additionally, students in general health education courses may be different from other college students with regards to their possibly increased sensitivity to health issues. Lastly, our study sample may not truly represent the broader student population, nor be generalizable to other campus settings.

\section{Conclusion}

Despite these limitations, we believe that our findings strongly suggest the importance of targeting men for prevention of HPV infection and cervical cancer in women. Such programs should include the benefits of limiting the number sexual partners and using condoms. These efforts should approach HPV prevention in a holistic manner, comparable to women's campaigns. They should highlight college-men's pivotal role in HPV infection in order to promote male empowerment and active involvement in HPV and cancer primary prevention efforts.

\section{References}

Auvinen, E., Niemi, M., Malm, C., Zalliacus, R., Trontti, A., Fingerross, R., et al. (2006). High prevalence of HPV among female students in Finland. Scandinavia Journal of Infectious Diseases, 37, 873-876.

Bandura, A. (1986). Social foundations of thought and action: A social cognitive theory. Englewood Cliffs, NJ: Prentice Hall.

Baseman, J., \& Koutsky, L. (2005). The epidemiology of human papillomavirus infections. Journal of Clinical Virology, 32, s16-s24.

Burk, R., Ho, G., Beardsley, L., Lempa M., Peters, M., \& Bierman, R. (1996). Sexual behavior and partner characteristics are the predominant risk factors for genital human papillomavirus infection in young women. Journal of Infectious Diseases, 174, 679-689.

Castellsague, X., Bosch, X., \& Munoz, N. (2001). The male role in cervical cancer. Salud Pública de México, 45, s345-s353.

Cecil, H., Pinkerton, S., \& Bogart, L. (1999). Perceived benefits and barriers associated with the female condom among African-American adults. Journal of Health Psychology, 4, 165-175.

Franceschi, S., Castellsague, X., Dal Maso, L., Smith, J., Plummer, M., Ngelangel, M., et al. (2001). Prevalence and determinants of human papillomavirus genital infection in men. British Journal of Cancer, 86, 705-711.

Franco, E., Schlecht, N., \& Saslow, D. (2003). The epidemiology of cervical cancer. The Cancer Journal. 9, 348-359.

Glanz, K., Lewis, F., \& Rimer, B. (2002). Health behavior and health education: Theory, research and practice. San Francisco: Jossey-Bass.

Hippelainen, M., Syrjanen S., \& Hippelainen, MI. (1993). Prevalence and risk factors of genital human papillomavirus (HVP) infections in healthy males: A study of Finish conscripts. Sexually Transmitted Diseases, 20, 321-328.

Koutsky, L. (1997). Epidemiology of genital human papillomavirus infection. American Journal of Medicine, 102, 3-8.

Lambert, E. (2001). College student's knowledge of human papillomavirus and effectiveness of a brief educational intervention. Journal of American Board Family Practice, 14, 178-183. 
Lazcano-Ponce, E., Herrero, R., Munos, N., Hernandez-Avila, M., Salmeron, J., Leyva, A., et al. (2000). High prevalence of human papillomavirus infection in Mexican males. Sexually Transmitted Diseases. 28, 277-280.

Lopez, R., \& McMahan, S. (2007). College women's (18-24) perception of human papillomavirus (HPV) and cervical cancer. Californian Journal of Health Promotion, 5(3), 12-25.

McPartland, T., Weaver, S., \& Koutsky L. (2005). Men's perceptions and knowledge of human papillomavirus (HPV) infection and cervical cancer. Journal of American College Health, 53, 225-230.

Munoz, N., \& Bosch, X. (1997). Cervical cancer and human papillomavirus: Epidemiological evidence and perspectives for prevention. Salud Pública de México, 39, 274-282.

Schiffman M., \& Castle, P. E. (2003). Human papillomavirus: Epidemiology and public health. Archives of Pathology \& Laboratory Medicine, 127, 930-934.

Shin, H., Franceschi, S., Vaccarella, S., Roh, J., Ju, J., Oh, J., et al. (2004). Prevalence and determinants of genital infection with Papillomavirus, in female and male university students in Busan, South Korea. Journal of Infectious Diseases, 190, 468-476.

Svare, E., Kjaer, S., Worm, A., Osternlind, A., Meijer, C., \& Van den Brule, A. (2002). Risk factors for genital HPV DNA in men resemble those found in women: a study of male attendees at Danish STD clinic. Sexually Transmitted Infections, 78, 215-218.

Winer, R., Lee, S., Hughes, J., Adam, D., Kiviat, N., \& Koutsky, L. (2003). Genital human papillomavirus infection: Incidence and risk factors in a cohort of female university students. American Journal of Epidemiology, 157, 218-226.

Wilkstrom, A., Popescu, C., \& Forrslund, O. (2000). Asymptomatic penile HPV infection: A prospective study. International Journal of STD \& AIDS, 11, 80-84.

Yacobi, E., Tennant, C., Ferrante, J., Pal., N., \& Roetzheim, R. (1999). University students' knowledge and awareness of HPV. Preventive Medicine, 28, 535-541.

Zak-Place, J., \& Stern, M. (2004). Health belief factors and dispositional optimism as predictors of STD and HIV preventative behavior. Journal of American College Health, 52, 229-236. 
Author Information

Ricardo V. López, MPH

Department of Health Science

California State University, Fullerton

Sora Park Tanjasiri, DrPH, MPH*

Department of Health Science

California State University, Fullerton

P.O. Box 6870

Fullerton, CA 92834

(714) 278-4592; (714) 278-5317 fax

stanjasiri@,fullerton.edu

Shari McMahan, PhD

Department of Health Science

California State University, Fullerton

* corresponding author 


\section{Appendix A}

Table 1:

Demographics and Sexual Behavior Characteristics of College-aged Men ( $\mathrm{n}=100)$

\begin{tabular}{|c|c|}
\hline Characteristics & Percent \\
\hline \multicolumn{2}{|l|}{ Ethnic Distribution } \\
\hline White & 29.0 \\
\hline Latino/Hispanic-American & 28.0 \\
\hline Asian/Pacific Islander & 27.0 \\
\hline Black/African-American & 3.0 \\
\hline Middle-Eastern & 3.0 \\
\hline Other & 10.0 \\
\hline Mean Age & 19.53 (SD 1.5) \\
\hline \multicolumn{2}{|l|}{ Class Standing } \\
\hline Freshmen & 48.0 \\
\hline Sophomore & 31.0 \\
\hline Junior & 11.0 \\
\hline Senior & 9.0 \\
\hline Graduate & 1.0 \\
\hline \multicolumn{2}{|l|}{ Student Status } \\
\hline Full-Time & 98.0 \\
\hline Part-Time & 2.0 \\
\hline \multicolumn{2}{|l|}{ Living Situation } \\
\hline Living at Home with Guardians & 67.0 \\
\hline Living Independently & 32.0 \\
\hline \multicolumn{2}{|c|}{ In Relationship with Significant Other } \\
\hline No & 43.0 \\
\hline Yes & 57.0 \\
\hline \multicolumn{2}{|c|}{ Relationship Considered Monogamous* } \\
\hline Yes & 80.5 \\
\hline No & 19.5 \\
\hline \multicolumn{2}{|l|}{ Currently Sexually Active* } \\
\hline Yes & 41.7 \\
\hline No & 58.3 \\
\hline \multicolumn{2}{|l|}{ Sexually Active With Whom* } \\
\hline Women & 96.8 \\
\hline Both & 3.2 \\
\hline \multicolumn{2}{|c|}{ Number of Sexual Partner(s) in Last 12 Months } \\
\hline 0 & 31.3 \\
\hline 1 & 32.3 \\
\hline $2-3$ & 24.0 \\
\hline $4-5$ & 8.3 \\
\hline $5+$ & 4.1 \\
\hline \multicolumn{2}{|c|}{ Wore Condoms in Last Sexual Encounter* } \\
\hline No & 43.5 \\
\hline Yes & 56.5 \\
\hline \multicolumn{2}{|c|}{ How Often Do They Wear Condoms* } \\
\hline Not at all & 38.1 \\
\hline All of the Time & 61.9 \\
\hline
\end{tabular}

* Calculated only for men who were sexually active 


\section{Appendix B}

Table 2:

College-aged Men's Knowledge of HPV and Cervical Cancer $(\mathrm{n}=100)$

\begin{tabular}{|c|c|c|}
\hline Variable & Answer & \% Correct \\
\hline 1. HPV can cause Herpes & False & 44.0 \\
\hline 2. Genital Warts are Caused by HPV & True & 57.0 \\
\hline 3. HPV can cause cervical cancer & True & 69.0 \\
\hline 4. If a woman's Pap smear is normal, she does not have HPV & False & 53.0 \\
\hline 5. Changes in a Pap smear may indicate that a woman has HPV & True & 65.0 \\
\hline 6. Genital warts are caused by the herpes virus & False & 25.0 \\
\hline 7. Pap smears will almost always detect HPV & False & 58.0 \\
\hline 8. HPV can be passed from the mother to her baby during birth & True & 58.0 \\
\hline 9. A negative test for HPV means that you do not have HPV & False & 44.0 \\
\hline 10. A vaccine exists to prevent HPV infection & True & 36.0 \\
\hline $\begin{array}{l}\text { 11. Most people with genital HPV have no visible signs or } \\
\text { symptoms. }\end{array}$ & True & 75.0 \\
\hline $\begin{array}{l}\text { 12. Having one type of HPV means that you cannot acquire new } \\
\text { types }\end{array}$ & False & 81.0 \\
\hline $\begin{array}{l}\text { 13. I can transmit HPV to my partner(s) even if I have no HPV } \\
\text { symptoms }\end{array}$ & True & 79.0 \\
\hline
\end{tabular}

1 Less than $9 \%$ of missing values all of the questions in the knowledge scale. Missing values were replaced by the mean values of all other values. 


\section{Appendix C}

Table 3:

Unadjusted Odd Ratios of HBM constructs with Intent to Wear Condoms (Predictor 1) and Intent to Reduce Number of Sex Partners (Predictor 2)

\begin{tabular}{|l|c|c|c|c|c|c|}
\hline \multirow{2}{*}{ Outcome Variables } & \multicolumn{3}{|c|}{ Predictor 1 } & \multicolumn{3}{|c|}{ Predictor 2 } \\
\cline { 2 - 7 } & $\mathrm{N}$ & $\mathrm{OR}$ & $(95 \% \mathrm{CI})$ & $\mathrm{N}$ & $\mathrm{OR}$ & $(95 \% \mathrm{CI})$ \\
\hline $\begin{array}{l}\text { Self-Efficacy } \\
\text { (Likely) }\end{array}$ & 47 & $24.44^{*}$ & $(6.13-97.48)$ & 26 & 0.83 & $(0.28-2.45)$ \\
\hline Perceived Benefits & 41 & 1.22 & $(0.37-4.08)$ & 33 & $3.77^{*}$ & $(1.16-12.25)$ \\
)
\end{tabular}

* Significant at the $\mathrm{p}<0.05$ level

** Of those that stated they were in a relationship 